TRANSACTIONS OF THE

AMERICAN MATHEMATICAL SOCIETY

Volume 359, Number 6, June 2007, Pages 2463-2481

S 0002-9947(07)04273-0

Article electronically published on January 19, 2007

\title{
HARNACK INEQUALITY FOR NON-DIVERGENCE FORM OPERATORS ON STRATIFIED GROUPS
}

\author{
ANDREA BONFIGLIOLI AND FRANCESCO UGUZZONI
}

\begin{abstract}
We prove lower bounds for the fundamental solutions of the nondivergence form operators$$
\sum_{i, j} a_{i, j}(x, t) X_{i} X_{j}-\partial_{t} \quad \text { and } \quad \sum_{i, j} a_{i, j}(x) X_{i} X_{j},
$$

where the $X_{i}$ 's are Hörmander vector fields generating a stratified group $\mathbb{G}$ and $\left(a_{i, j}\right)_{i, j}$ is a positive-definite matrix with Hölder continuous entries. We then prove an invariant Harnack inequality for such operators. As a byproduct we also study some relevant properties of the Green functions on bounded domains.
\end{abstract}

\section{INTRODUCTION AND MAIN RESULTS}

The aim of this paper is to prove an invariant Harnack inequality for the nondivergence form operator

$$
\mathcal{H}=\sum_{i, j=1}^{m} a_{i, j}(x, t) X_{i} X_{j}-\partial_{t} .
$$

Here $\left\{X_{i}\right\}_{i}$ is a stratified system of vector fields in $\mathbb{R}^{N}$ and $\left(a_{i, j}\right)_{i, j \leq m}$ is a positivedefinite matrix with Hölder continuous entries. Our main step in deriving this inequality is to establish a Gaussian lower bound for the fundamental solution of $\mathcal{H}$. This bound also gives an estimate from below of the fundamental solution for the associated stationary operator. Our main results are contained in Theorem 1.1. Theorem 1.2 and Theorem 1.3 below. As a byproduct we also study some relevant properties of the Green function related to $\mathcal{H}$ on bounded domains.

Before stating our results and describing our approach, we would like to give some motivation for our research. Second order linear and nonlinear PDE's of subelliptic type arise in many different settings: geometric theory of several complex variables, curvature problems for CR-manifolds, sub-Riemannian geometry, diffusion processes, control theory, human vision, see e.g., 1, 14, 15, 20, 21, 24, 26, 31, 32, 33, 36, 37, 38. The underlying algebraic structures of all these equations are homogeneous stratified groups $\mathbb{G}$ (see the definition below). Several results concerning the divergence-form counterpart of (1.1) are present in the literature, both for linear and quasi-linear equations, see e.g., [8, 9, 10, 11, 13, 18, 25, 28, 29, 30, 35, 39. On the contrary, to the authors' knowledge, very few papers are devoted to nondivergence form operators of the above type; see [3, 6, 17, 12, 39. We would like to

Received by the editors September 26, 2003.

2000 Mathematics Subject Classification. Primary 35B05, 35A08, 43A80; Secondary 35H20, 35J70.

This work was supported by the University of Bologna, Funds for selected research topics. 
stress that operators exactly in this form arise in studying motion by Levi curvature of hypersurfaces of $\mathbb{C}^{n+1}$ [20, 31. Our aim in this paper is to provide a new contribution to the study of non-divergence form operators (1.1). Indeed, our results here are a step in a wider project aimed to apply analysis on stratified groups to the study of fully non-linear PDE's of sub-elliptic type, such as the Levi-curvature equation and its parabolic counterpart, for which operators like (1.1) arise as linearizations.

We now briefly describe our approach. The main tool in proving the Harnack inequality is a suitable adaptation of the method by Krylov and Safanov, also used by Fabes and Stroock [16] for classical parabolic equations and by Kusuoka and Stroock [22, 23] for parabolic equations modelled on a sum of squares of smooth vector fields satisfying the Hörmander condition. In the latter paper, this method has been applied in a sub-elliptic context similar to ours, jointly with probabilistic results. We do not use probabilistic techniques, but we make a crucial use of the existence of the relevant Green function on a suitable family of sets. More precisely, we construct the Green function for the operator (1.1) on cylindrical domains which are regular for the Dirichlet problem related to $\mathcal{H}$, and we show that an arbitrary cylindrical domain can be approximated from the inside by a sequence of $\mathcal{H}$-regular domains. Some needed uniform properties of such approximation are non-trivial and require a careful potential theory investigation, which is the object of the forthcoming paper 27. We remark that the existence of the Green function $G$ on such approximating domains is indeed sufficient in order to adapt the cited Krylov and Safanov method, once some well-behaved invariant estimates of $G$ are available. We prove such invariant estimates of $G$ as a consequence of the good behavior of our approximation with respect to the underlying Carnot-Carathéodory metric structure (see Theorem 4.3). We finally would like to stress that the proof of the Gaussian bound from below of the fundamental solution for $\mathcal{H}$ (from which the estimates of $G$ are derived) is based on some non-trivial uniform estimates for the relevant frozen operators and relies on the work made in [2, 4, 5] (see also [35]).

We now introduce some notation and then state our main results. We start by giving the definition of a Carnot group. Let $\circ$ be an assigned Lie group law on $\mathbb{R}^{N}$. Suppose $\mathbb{R}^{N}$ is endowed with a homogeneous structure by a given family of Lie group automorphisms $\left\{\delta_{\lambda}\right\}_{\lambda>0}$ (called dilations) of the form $\delta_{\lambda}(x)=\delta_{\lambda}\left(x^{(1)}, \ldots, x^{(r)}\right)=$ $\left(\lambda x^{(1)}, \ldots, \lambda^{r} x^{(r)}\right)$. Here $x^{(i)} \in \mathbb{R}^{N_{i}}$ for $i=1, \ldots, r$ and $N_{1}+\cdots+N_{r}=N$. We denote by $\mathfrak{g}$ the Lie algebra of $\left(\mathbb{R}^{N}, \circ\right)$, i.e., the Lie algebra of left-invariant vector fields on $\mathbb{R}^{N}$. For $i=1, \ldots, N_{1}$, let $X_{i}$ be the (unique) vector field in $\mathfrak{g}$ that agrees at the origin with $\partial / \partial x_{i}^{(1)}$. We make the following assumption: the Lie algebra generated by $X_{1}, \ldots, X_{N_{1}}$ is the whole $\mathfrak{g}$. With the above hypotheses, we call $\mathbb{G}=\left(\mathbb{R}^{N}, \circ, \delta_{\lambda}\right)$ a Carnot group. We also say that $\mathbb{G}$ is of step $r$ and has $m:=N_{1}$ generators. In the literature (see e.g., [17, 19, 34, 38]) a Carnot group (or stratified group) $\mathbb{H}$ is defined as a connected and simply connected Lie group whose Lie algebra $\mathfrak{h}$ admits a stratification $\mathfrak{h}=\mathfrak{G}_{1} \oplus \cdots \oplus \mathfrak{G}_{r}$ with $\left[\mathfrak{G}_{1}, \mathfrak{G}_{i}\right]=\mathfrak{G}_{i+1}$, $\left[\mathfrak{G}_{1}, \mathfrak{G}_{r}\right]=\{0\}$. The two definitions are equivalent up to isomorphism. We denote by $Q=\sum_{j=1}^{r} j N_{j}$ the homogeneous dimension of $\mathbb{G}$. If $Q \leq 3$, then $\mathbb{G}$ is the ordinary Euclidean group $\left(\mathbb{R}^{Q},+\right)$. Hence, throughout the paper, we shall always assume $Q \geq 4$. In the sequel, $d$ will denote the Carnot-Carathéodory control distance associated to the family of vector fields $X_{1}, \ldots, X_{m}$. We recall that this distance is equivalent to any quasi-distance induced by a homogeneous norm on $\mathbb{G}$. 
Hereafter, we also denote $d(x, 0)$ simply by $d(x)$ and use the notation $B_{d}(x, r)$ for the $d$-ball of center $x \in \mathbb{G}$ and radius $r>0$.

Let us fix the parameters $\Lambda>1, k>0$ and $\alpha \in(0,1]$. Throughout the paper, we shall denote by $\mathbf{c}$ any positive constant depending only on these parameters (and on the structure of $\mathbb{G}$ ). Moreover, we shall use the notation $\mathbf{c}\left(f_{1}, \ldots, f_{n}\right)$ if $\mathbf{c}$ also depends on $f_{1}, \ldots, f_{n}$. Let $A=\left(a_{i, j}\right)_{i, j \leq m}$ be a function defined on $\mathbb{R}^{N+1}$ and taking values in the symmetric $m \times m$ matrices. Throughout the paper we shall suppose that $A$ satisfies the following conditions:

$$
\begin{array}{r}
\Lambda^{-1}|\eta|^{2} \leq\langle A(x, t) \eta, \eta\rangle \leq \Lambda|\eta|^{2} \quad \forall \eta \in \mathbb{R}^{m}, \\
\left|a_{i, j}(x, t)-a_{i, j}\left(x^{\prime}, t^{\prime}\right)\right| \leq k\left(d\left(x, x^{\prime}\right)^{\alpha}+\left|t-t^{\prime}\right|^{\alpha / 2}\right),
\end{array}
$$

for every $(x, t),\left(x^{\prime}, t^{\prime}\right) \in \mathbb{R}^{N+1}$. Hereafter, we use the notation $z=(x, t)$ and $\zeta=(\xi, \tau)$ for the point of $\mathbb{R}^{N+1}(x, \xi \in \mathbb{G}, t, \tau \in \mathbb{R})$. We denote by $\mathcal{H}$ the parabolic-type operator

$$
\mathcal{H}=\sum_{i, j=1}^{m} a_{i, j}(x, t) X_{i} X_{j}-\partial_{t}
$$

The main result of this paper is the following invariant Harnack inequality for $\mathcal{H}$.

Theorem 1.1. Let $R_{0}>0,0<h_{1}<h_{2}<1$ and $\gamma \in(0,1)$. There exists a positive constant $M=\mathbf{c}\left(h_{1}, h_{2}, \gamma, R_{0}\right)$ such that

$$
\max _{\overline{B_{d}\left(\xi_{0}, \gamma R\right)} \times\left[\tau_{0}-h_{2} R^{2}, \tau_{0}-h_{1} R^{2}\right]} u \leq M u\left(\xi_{0}, \tau_{0}\right)
$$

for every $\left(\xi_{0}, \tau_{0}\right) \in \mathbb{R}^{N+1}, R \in\left(0, R_{0}\right]$ and every $u \in \Gamma^{2}\left(B_{d}\left(\xi_{0}, R\right) \times\left(\tau_{0}-R^{2}, \tau_{0}\right)\right) \cap$ $C\left(\overline{B_{d}\left(\xi_{0}, R\right)} \times\left[\tau_{0}-R^{2}, \tau_{0}\right]\right)$ satisfying $\mathcal{H} u=0, u \geq 0$ in $B_{d}\left(\xi_{0}, R\right) \times\left(\tau_{0}-R^{2}, \tau_{0}\right)$.

We have used the notation $\Gamma^{2}(\Omega)$ to denote the space of continuous functions, defined on an open set $\Omega \subseteq \mathbb{R}^{N+1}$, which have continuous Lie-derivatives along the vector fields $X_{1}, \ldots, X_{m}$ up to second order and continuous derivative along $\partial_{t}$ (up to first order). In the sequel we also denote by $\Gamma^{\beta}(\Omega)(0<\beta<1)$ the Hölder space of functions $v: \Omega \rightarrow \mathbb{R}$ such that

$$
\|u\|_{\Gamma^{\beta}(\Omega)}:=\sup _{\Omega}|u|+\sup _{(x, t) \neq\left(x^{\prime}, t^{\prime}\right) \in \Omega} \frac{\left|u(x, t)-u\left(x^{\prime}, t^{\prime}\right)\right|}{d\left(x, x^{\prime}\right)^{\beta}+\left|t-t^{\prime}\right|^{\beta / 2}}<\infty .
$$

Moreover, $\Gamma^{2+\beta}(\Omega)$ will denote the space of functions $u: \Omega \rightarrow \mathbb{R}$ which belong to $\Gamma^{\beta}(\Omega)$ together with any Lie-derivative along the vector fields $X_{1}, \ldots, X_{m}$ up to second order, and along $\partial_{t}$ up to first order. Analogously one defines $\Gamma_{\text {loc }}^{2+\beta}(\Omega)$. The fundamental solution $\Gamma$ of the operator $\mathcal{H}$ has been constructed in [3]: we recall some needed properties of $\Gamma$ in Theorem $A$ at the end of the section. In particular, in [3] we proved a Gaussian estimate of $\Gamma$ from above. As a main step in the proof of Theorem 1.1. here we also prove that a pointwise estimate from below of the same kind holds.

Theorem 1.2. For every $T>0$, there exists a positive constant $\mathbf{c}(T)$ such that

$$
\begin{aligned}
& \qquad(x, t ; \xi, \tau) \geq \mathbf{c}(T)^{-1}(t-\tau)^{-Q / 2} \exp \left(-\mathbf{c} d^{2}(x, \xi) /(t-\tau)\right), \\
& \text { for } 0<t-\tau \leq T \text { and } x, \xi \in \mathbb{R}^{N} \text {. }
\end{aligned}
$$

We now briefly turn to consider the stationary operator

$$
\mathcal{L}=\sum_{i, j=1}^{m} a_{i, j}(x) X_{i} X_{j}
$$


on $\mathbb{R}^{N}$ (here the coefficients $a_{i, j}$ are supposed as in (1.2) but not depending on $t$ ). Clearly from Theorem 1.1, an invariant Harnack inequality for $\mathcal{L}$ follows straightforwardly. Moreover, by integrating the fundamental solution $\Gamma$ of $\mathcal{L}-\partial_{t}$ over the variable $t$, thanks to suitable long-time estimates of $\Gamma$ established in $[3$, one can construct local fundamental solutions $\gamma$ for $\mathcal{L}$ (see Theorem $\mathrm{C}$ below) if the dimension $m$ of the first layer of the stratification of $\mathfrak{g}$ is strictly greater than two.

Finally, by means of Theorem [1.2 we easily find an estimate of $\gamma$ from below.

Theorem 1.3. With the hypotheses and notation of Theorem $\mathrm{C}$ below, there exists a positive constant $c$ such that

$$
\gamma(x, \xi) \geq c^{-1} d(x, \xi)^{2-Q}
$$

for every $x, \xi \in \mathbb{R}^{N}$ such that $0<d(x, \xi)<1$.

We end the section by recalling some needed properties of $\Gamma$ and $\gamma$ proved in $[3$.

Theorem A. (i) $\Gamma$ is a continuous function away from the diagonal of $\mathbb{R}^{N+1} \times$ $\mathbb{R}^{N+1}$. Moreover, for every fixed $\zeta \in \mathbb{R}^{N+1}, \Gamma(\cdot ; \zeta) \in \Gamma_{\text {loc }}^{2+\alpha}\left(\mathbb{R}^{N+1} \backslash\{\zeta\}\right)$ and we have $\mathcal{H}(\Gamma(\cdot ; \zeta))=0$ in $\mathbb{R}^{N+1} \backslash\{\zeta\}$.

(ii) $\Gamma(x, t ; \xi, \tau)=0$ for $t \leq \tau$. Moreover, for $0<t-\tau \leq T$, the following estimate holds:

$$
0 \leq \Gamma(x, t ; \xi, \tau) \leq \mathbf{c}(T)(t-\tau)^{-Q / 2} \exp \left(-\mathbf{c}^{-1} d^{2}(x, \xi) /(t-\tau)\right) .
$$

(iii) For every $\psi \in C_{0}^{\infty}\left(\mathbb{R}^{N+1}\right)$, the function $w(z)=\int_{\mathbb{R}^{N+1}} \Gamma(z ; \zeta) \psi(\zeta) \mathrm{d} \zeta$ belongs to the class $\Gamma_{\text {loc }}^{2+\alpha}\left(\mathbb{R}^{N+1}\right)$, and we have $\mathcal{H} w=-\psi$ in $\mathbb{R}^{N+1}$.

(iv) Let $\mu \geq 0$ and $T_{2}>T_{1}$ be such that $\left(T_{2}-T_{1}\right) \mu$ is small enough. Then, for every $f \in \Gamma^{\beta}\left(\mathbb{R}^{N} \times\left[T_{1}, T_{2}\right]\right.$ ) (where $0<\beta \leq \alpha$ ) and $g \in C\left(\mathbb{R}^{N}\right)$ satisfying the growth condition $|f(x, t)|,|g(x)| \leq c \exp \left(\mu d^{2}(x)\right)$ for some constant $c>0$, the function

$$
\begin{gathered}
u(x, t)=\int_{\mathbb{R}^{N}} \Gamma\left(x, t ; \xi, T_{1}\right) g(\xi) \mathrm{d} \xi+\int_{\mathbb{R}^{N} \times\left[T_{1}, t\right]} \Gamma(x, t ; \xi, \tau) f(\xi, \tau) \mathrm{d} \xi \mathrm{d} \tau \\
\left(x \in \mathbb{R}^{N}, t \in\left(T_{1}, T_{2}\right]\right) \text { belongs to the class } \\
\Gamma_{\text {loc }}^{2+\beta}\left(\mathbb{R}^{N} \times\left(T_{1}, T_{2}\right)\right) \cap C\left(\mathbb{R}^{N} \times\left[T_{1}, T_{2}\right]\right) .
\end{gathered}
$$

Moreover, $u$ is a solution to the Cauchy problem $\mathcal{H} u=-f$ in $\mathbb{R}^{N} \times\left(T_{1}, T_{2}\right)$, $u\left(\cdot, T_{1}\right)=g$.

Theorem B. Let $\Omega$ be a bounded open subset of $\mathbb{R}^{N+1}$ and let $t_{0} \in \mathbb{R}$. If $u \in \Gamma^{2}(\Omega)$, $\mathcal{H} u \geq 0$ in $\Omega \cap\left\{t<t_{0}\right\}$, and $\limsup u \leq 0$ in $\partial \Omega \cap\left\{t \leq t_{0}\right\}$, then $u \leq 0$ in $\Omega \cap\left\{t<t_{0}\right\}$.

We shall also use the following version of the weak maximum principle in infinite strips which easily follows from Theorem B.

Corollary 1.4. Let $u \in \Gamma^{2}\left(\mathbb{R}^{N} \times\left(T_{1}, T_{2}\right)\right)$ be such that $\mathcal{H} u \geq 0$ in $\mathbb{R}^{N} \times\left(T_{1}, T_{2}\right)$ and $\lim \sup u \leq 0$ in $\mathbb{R}^{N} \times\left\{T_{1}\right\}$ and at infinity. Then $u \leq 0$ in $\mathbb{R}^{N} \times\left(T_{1}, T_{2}\right)$.

Theorem C. Suppose that $m>2$. For every fixed bounded open set $\Omega \subset \mathbb{R}^{N}$, there exists a function $\gamma$ defined on $\mathbb{R}^{N} \times \mathbb{R}^{N}$ with the following properties:

(i) $\gamma$ is a continuous function away from the diagonal of $\mathbb{R}^{N} \times \mathbb{R}^{N}$. Moreover, for every fixed $\xi \in \mathbb{R}^{N}, \gamma(\cdot, \xi) \in \Gamma_{\text {loc }}^{2+\alpha}\left(\mathbb{R}^{N} \backslash\{\xi\}\right)$, and we have $\mathcal{L}(\gamma(\cdot, \xi))=0$ in $\Omega \backslash\{\xi\}$. 
(ii) For every compact set $K \Subset \mathbb{R}^{N}$ there exists a positive constant $c$ such that

$$
0 \leq \gamma(x, \xi) \leq c\left(1+d(x, \xi)^{2-Q}\right), \quad \xi \in K, x \in \mathbb{R}^{N} .
$$

(iii) For every $\psi \in C_{0}^{\infty}\left(\mathbb{R}^{N}\right)$, the function $w(x)=\int_{\mathbb{R}^{N}} \gamma(x ; \xi) \psi(\xi) \mathrm{d} \xi$ belongs to the class $\Gamma_{\text {loc }}^{2+\alpha}\left(\mathbb{R}^{N}\right)$, and we have $\mathcal{L} w=-\psi$ in $\Omega$.

The paper is organized as follows. In Section 2 we prove the lower bounds of Theorem 1.2 and Theorem [1.3. In Section 3 we study the Green functions on $\mathcal{H}$-regular cylinders. In Section 4 we prove Theorem 1.1 under the additional assumption that the coefficients $a_{i, j}$ of $\mathcal{H}$ are smooth. Finally in Section 5 we complete the proof of the Harnack inequality of Theorem 1.1 in the general case.

\section{THE LOWER BOUNDS OF $\Gamma$ AND $\gamma$}

The aim of this section is to prove Theorem 1.2 and Theorem 1.3

We first prove the following reproduction property of $\Gamma$.

Proposition 2.1. We have

$$
\Gamma(x, t ; \xi, \tau)=\int_{\mathbb{R}^{N}} \Gamma(x, t ; y, s) \Gamma(y, s ; \xi, \tau) \mathrm{d} y,
$$

for $t>s>\tau$ and $x, \xi \in \mathbb{R}^{N}$.

Proof. We fix $\xi, \tau$ and $s$ as above, and we set $u=\int_{\mathbb{R}^{N}} \Gamma(\cdot ; y, s) \Gamma(y, s ; \xi, \tau) \mathrm{d} y$ and $v=\Gamma(\cdot ; \xi, \tau)$. From Theorem A(iv) it follows that (for $\left.T_{2}>T_{1}=s\right) u \in$ $\Gamma_{\text {loc }}^{2+\alpha}\left(\mathbb{R}^{N} \times\left(s, T_{2}\right)\right) \cap C\left(\mathbb{R}^{N} \times\left[s, T_{2}\right]\right), \mathcal{H} u=0$ in $\mathbb{R}^{N} \times\left(s, T_{2}\right)$ and $u(\cdot, s)=v(\cdot, s)$ (note that $v(\cdot, s)$ is continuous and bounded, by Theorem $\mathrm{A}(\mathrm{i})-(\mathrm{ii})$ ). On the other hand, again from Theorem A(i), we know that $v \in \Gamma_{\text {loc }}^{2+\alpha}\left(\mathbb{R}^{N+1} \backslash\{(\xi, \tau)\}\right), \mathcal{H} v=0$ in $\mathbb{R}^{N+1} \backslash\{(\xi, \tau)\}$. Thus, we only need to show that

$$
\sup _{s<t<T_{2}, d(x)>R}|u(x, t)-v(x, t)| \longrightarrow 0, \quad \text { as } R \rightarrow \infty,
$$

in order to get $u=v$ in $\mathbb{R}^{N} \times\left(s, T_{2}\right)$ from the weak maximum principle in Corollary 1.4. Let us prove (2.1). Denoting by $\Gamma_{0}$ the fundamental solution of the model heat operator $\sum_{j=1}^{m} X_{j}^{2}-\partial_{t}$ on $\mathbb{G}$ and using the reproduction property and the Gaussian bounds of $\Gamma_{0}$ (see e.g., [38; see also 2]) along with (1.3), we obtain

$$
\begin{aligned}
u(x, t) & \leq \mathbf{c}\left(T_{2}, \tau\right) \int_{\mathbb{R}^{N}} \Gamma_{0}\left(y^{-1} \circ x, \mathbf{c}_{1}(t-s)\right) \Gamma_{0}\left(\xi^{-1} \circ y, \mathbf{c}_{1}(s-\tau)\right) \mathrm{d} y \\
& =\mathbf{c}\left(T_{2}, \tau\right) \Gamma_{0}\left(\xi^{-1} \circ x, \mathbf{c}_{1}(t-\tau)\right) \leq \mathbf{c}\left(T_{2}, \tau\right)(t-\tau)^{-Q / 2} \exp \left(-\frac{d^{2}(x, \xi)}{\mathbf{c}(t-\tau)}\right)
\end{aligned}
$$

for every $(x, t) \in \mathbb{R}^{N} \times\left(s, T_{2}\right)$. We now use the estimate (1.3) of $v$, and we finally obtain

$$
\sup _{s<t<T_{2}, d(x)>R}(|u(x, t)|+|v(x, t)|) \leq \mathbf{c}\left(T_{2}, \tau, s\right) \sup _{d(x)>R} \exp \left(-\frac{d^{2}(x, \xi)}{\mathbf{c}\left(T_{2}, \tau\right)}\right),
$$

which gives (2.1).

The main step in the proof of Theorem 1.2 is the following lemma.

Lemma 2.2. There exists a positive constant $\delta$, only depending on the parameters $\Lambda, k, \alpha$ in (1.2) (and on the structure of $\mathbb{G}$ ), such that

$$
\Gamma(x, t ; \xi, \tau) \geq \mathbf{c}^{-1}(t-\tau)^{-Q / 2} \exp \left(-\mathbf{c} d^{2}(x, \xi) /(t-\tau)\right),
$$

if $0<t-\tau<\delta$ and $d^{2}(x, \xi) \leq \delta(t-\tau)|\log (t-\tau)|$. 
Proof. Let us denote by $\Gamma_{\zeta}$ the fundamental solution of the operator obtained from $\mathcal{H}$ by freezing the coefficients $a_{i, j}$ at the point $\zeta$. In [3, (2.15)], it was explicitly proved that

$$
\left|\Gamma(z ; \zeta)-\Gamma_{\zeta}(z ; \zeta)\right| \leq \mathbf{c}(T)(t-\tau)^{\frac{\alpha-Q}{2}} \exp \left(-\mathbf{c}^{-1} d^{2}(x, \xi) /(t-\tau)\right),
$$

for every $z=(x, t), \zeta=(\xi, \tau)$ such that $o<t-\tau \leq T$. Moreover $\Gamma_{\zeta}$ has a Gaussian bound from below, uniform in $\zeta \in \mathbb{R}^{N+1}$ :

$$
\Gamma_{\zeta}(x, t ; y, s) \geq \mathbf{c}(\Lambda)^{-1}(t-s)^{-Q / 2} \exp \left(-\mathbf{c}(\Lambda) d^{2}(x, y) /(t-s)\right), \quad \text { for } t>s
$$

(see [35] and [2, Theorem 2.5]). As a consequence, for $0<t-\tau \leq 1=T$, we get

$$
\begin{aligned}
& \Gamma(z ; \zeta) \geq \Gamma_{\zeta}(z ; \zeta)-\left|\Gamma(z ; \zeta)-\Gamma_{\zeta}(z ; \zeta)\right| \\
& \geq \mathbf{c}^{-1}(t-\tau)^{-\frac{Q}{2}} \exp \left(-\mathbf{c} \frac{d^{2}(x, \xi)}{t-\tau}\right)\left(1-\mathbf{c}_{1}(t-\tau)^{\frac{\alpha}{2}} \exp \left(\mathbf{c}_{1} \frac{d^{2}(x, \xi)}{t-\tau}\right)\right) .
\end{aligned}
$$

We now choose $\delta<\min \left\{1, \frac{\alpha}{4 \mathbf{c}_{1}},\left(2 \mathbf{c}_{1}\right)^{-4 / \alpha}\right\}$. If $0<t-\tau<\delta$ and $d^{2}(x, \xi) \leq$ $\delta(t-\tau)|\log (t-\tau)|$, we have

$$
\mathbf{c}_{1}(t-\tau)^{\frac{\alpha}{2}} \exp \left(\mathbf{c}_{1} d^{2}(x, \xi) /(t-\tau)\right) \leq \mathbf{c}_{1}(t-\tau)^{\frac{\alpha}{2}-\delta \mathbf{c}_{1}} \leq \mathbf{c}_{1}(t-\tau)^{\frac{\alpha}{4}} \leq \frac{1}{2} .
$$

Putting this estimate in (2.2), the proof is complete.

Proof of Theorem 1.2, Let $\delta$ be the constant in Lemma 2.2 and let us fix $T>$ $\delta, x, \xi \in \mathbb{R}^{N}$ and $0<t-\tau \leq T$. Let $k$ be the smallest integer greater than $\max \left\{T \delta^{-1}, 4 d^{2}(x, \xi)(\delta(t-\tau))^{-1}\right\}$ and let us set $\sigma=\frac{1}{4} \sqrt{\delta(t-\tau) /(k+1)}$. There exists a chain of points $x=x_{0}, x_{1}, \ldots, x_{k+1}=\xi$ (laying on a suitable $X$-subunit path which gives a $d$-geodesic connecting the points $x$ and $\xi)$ such that $d\left(x_{j}, x_{j+1}\right)=$ $d(x, \xi) /(k+1)$, for $j=0, \ldots, k$. Moreover, there exist real numbers $t=t_{0}, t_{1}, \ldots$, $t_{k+1}=\tau$ such that $t_{j}-t_{j+1}=(t-\tau) /(k+1)$ for $j=0, \ldots, k$. Using Proposition 2.1 repeatedly and following an argument in [16, we obtain

$$
\begin{aligned}
& \Gamma(x, t ; \xi, \tau)=\int_{\mathbb{R}^{N}} \Gamma\left(x, t ; y_{1}, t_{1}\right) \Gamma\left(y_{1}, t_{1} ; \xi, \tau\right) \mathrm{d} y_{1} \\
& =\int_{\left(\mathbb{R}^{N}\right)^{k}} \Gamma\left(x, t ; y_{1}, t_{1}\right) \Gamma\left(y_{1}, t_{1} ; y_{2}, t_{2}\right) \cdots \Gamma\left(y_{k}, t_{k} ; \xi, \tau\right) \mathrm{d} y_{1} \cdots \mathrm{d} y_{k} \\
& \geq \int_{B_{d}\left(x_{1}, \sigma\right) \times \cdots \times B_{d}\left(x_{k}, \sigma\right)} \Gamma\left(y_{0}, t_{0} ; y_{1}, t_{1}\right) \cdots \Gamma\left(y_{k}, t_{k} ; y_{k+1}, t_{k+1}\right) \mathrm{d} y_{1} \cdots \mathrm{d} y_{k}
\end{aligned}
$$

(here we have set $y_{0}=x, y_{k+1}=\xi$ ). We now observe that $0<t_{j}-t_{j+1}<\delta$ and $d^{2}\left(y_{j}, y_{j+1}\right) \leq \delta\left(t_{j}-t_{j+1}\right)$ if $d\left(x_{j}, y_{j}\right)<\sigma$. Indeed, by the definition of $k$ and of $\sigma$, we have $t_{j}-t_{j+1}=(t-\tau) /(k+1)<T / k<\delta$, and

$$
\begin{aligned}
d\left(y_{j}, y_{j+1}\right) & \leq d\left(y_{j}, x_{j}\right)+d\left(x_{j}, x_{j+1}\right)+d\left(x_{j+1}, y_{j+1}\right) \\
& <2 \sigma+d(x, \xi) /(k+1)<2 \sigma+\frac{\sqrt{k \delta(t-\tau)}}{2(k+1)}<\sqrt{\frac{\delta(t-\tau)}{(k+1)}} .
\end{aligned}
$$

Thus, we can apply Lemma 2.2 and obtain

$$
\begin{aligned}
\Gamma(x, t ; \xi, \tau) \geq & \left(\mathbf{c}^{-1}((t-\tau) /(k+1))^{-\frac{Q}{2}}\right)^{k+1} \\
& \times \int_{B_{d}\left(x_{1}, \sigma\right) \times \cdots \times B_{d}\left(x_{k}, \sigma\right)} \exp \left(-\mathbf{c} \sum_{j=0}^{k} \frac{d^{2}\left(y_{j}, y_{j+1}\right)}{t_{j}-t_{j+1}}\right) \mathrm{d} y_{1} \cdots \mathrm{d} y_{k} \\
\geq & \mathbf{c}^{-1}\left(\frac{t-\tau}{k+1}\right)^{-\frac{Q}{2}(k+1)}\left(\left|B_{d}(0,1)\right| \sigma^{Q}\right)^{k} \exp (-\mathbf{c} k) \geq \mathbf{c}^{-1}(t-\tau)^{-\frac{Q}{2}} \exp (-\mathbf{c} k),
\end{aligned}
$$


by the definition of $\sigma$. Now, if $d^{2}(x, \xi) \geq T(t-\tau) / 4$, from the definition of $k$ it follows that $k<8 \delta^{-1} d^{2}(x, \xi) /(t-\tau)$ and then

$$
\Gamma(x, t ; \xi, \tau) \geq \mathbf{c}^{-1}(t-\tau)^{-\frac{Q}{2}} \exp \left(-\mathbf{c} d^{2}(x, \xi) /(t-\tau)\right) .
$$

On the other hand, if $d^{2}(x, \xi)<T(t-\tau) / 4$, the definition of $k$ gives $k<2 T / \delta$ and then

$$
\Gamma(x, t ; \xi, \tau) \geq \mathbf{c}(T)^{-1}(t-\tau)^{-\frac{Q}{2}} \geq \mathbf{c}(T)^{-1}(t-\tau)^{-\frac{Q}{2}} \exp \left(-\mathbf{c} d^{2}(x, \xi) /(t-\tau)\right) .
$$

This completes the proof.

Proof of Theorem 1.3. We fix $\varphi \in C_{0}^{\infty}\left(\mathbb{R}^{N}\right)$ such that $0 \leq \varphi \leq 1$ and $\varphi \equiv 1$ in $\Omega$. We set

$$
\left(\widetilde{a}_{i, j}(x)\right)_{i, j}=\widetilde{A}(x)=\varphi(x) A(x)+(1-\varphi(x))(4 \Lambda)^{-1} \mathbb{I}_{m}
$$

(where $\mathbb{I}_{m}$ denotes the $m \times m$ identity matrix), and we define $\widetilde{\mathcal{H}}=\widetilde{\mathcal{L}}-\partial_{t}=$ $\sum_{i, j=1}^{m} \widetilde{a}_{i, j}(x) X_{i} X_{j}-\partial_{t}$. Then $\widetilde{A}$ satisfies (1.2), with $\widetilde{\Lambda}=4 \Lambda, \widetilde{\alpha}=\alpha, \widetilde{k}=\mathbf{c}$ (if $\varphi$ is suitably chosen). Thus we can apply Theorem 1.2 to the fundamental solution $\widetilde{\Gamma}$ of $\widetilde{\mathcal{H}}$. Moreover, in 3 , we have shown that the above alteration of the coefficients enables us to obtain suitable long-time estimates of $\widetilde{\Gamma}$ and, as a consequence, to prove that $\gamma(x, \xi)=\int_{0}^{\infty} \widetilde{\Gamma}(x, t ; \xi, 0) \mathrm{d} t$ is a fundamental solution of $\widetilde{\mathcal{L}}$. Furthermore $\gamma$ is also a local fundamental solution of $\mathcal{L}$ on $\Omega$ since $\mathcal{L}=\widetilde{\mathcal{L}}$ in $\Omega$ by definition. Finally, from Theorem 1.2 we obtain

$$
\begin{aligned}
\gamma(x, \xi) & \geq \int_{0}^{1} \widetilde{\Gamma}(x, t ; \xi, 0) \mathrm{d} t \geq \mathbf{c}^{-1} \int_{0}^{1} t^{-Q / 2} \exp \left(-\mathbf{c} d(x, \xi)^{2} / t\right) \mathrm{d} t \\
& =\mathbf{c}^{-1} d(x, \xi)^{2-Q} \int_{\mathbf{c} d(x, \xi)^{2}}^{\infty} s^{(Q-4) / 2} \exp (-s) \mathrm{d} s \geq \mathbf{c}^{-1} d(x, \xi)^{2-Q},
\end{aligned}
$$

if $0<d(x, \xi)<1$.

\section{GREen FunCtions For $\mathcal{H}$ ON INFINITE CYLINDERS}

Throughout Sections 3 and 4 . we shall make the qualitative assumption that the coefficients $a_{i, j}$ of $\mathcal{H}$ are smooth. In Section 5, we shall turn back to Hölder continuous coefficients $a_{i, j}$ and complete the proof of our main Theorem 1.1, by an approximation argument.

We shall say that a bounded cylinder $D=A \times\left(T_{1}, T_{2}\right) \subseteq \mathbb{R}^{N+1}$ is $\mathcal{H}$-regular if for every continuous function $\varphi$ on the parabolic boundary $\partial_{p} D=\left(\partial A \times\left[T_{1}, T_{2}\right]\right) \cup$ $\left(\bar{A} \times\left\{T_{1}\right\}\right)$ there exists a (unique) solution $u_{\varphi}$ to

$$
u \in \Gamma^{2}(D) \cap C\left(D \cup \partial_{p} D\right), \quad \mathcal{H} u=0 \text { in } D, \quad u=\varphi \text { in } \partial_{p} D .
$$

We then denote by $\left\{\mu_{z}^{D}\right\}_{z \in D}$ the relevant family of $\mathcal{H}$-harmonic measures (supported on $\left.\partial_{p} D\right)$ so that $u_{\varphi}(z)=\int \varphi(\zeta) \mathrm{d} \mu_{z}^{D}(\zeta)$. Finally, for convenience of notation, we shall also say that a bounded open set $A \subseteq \mathbb{R}^{N}$ is $\mathcal{H}$-regular if, for any $T_{1}<T_{2}$, the cylinder $A \times\left(T_{1}, T_{2}\right)$ is $\mathcal{H}$-regular. We remark that any bounded open set $A \subseteq \mathbb{R}^{N}$ can be approximated by a sequence of $\mathcal{H}$-regular domains (see [27]; see also Lemmas 4.1 and 5.11). The aim of this section is to prove the following result. 
Theorem 3.1. Let $A \subseteq \mathbb{R}^{N}$ be an $\mathcal{H}$-regular domain. Then there exists a Green function $G=G^{A}$ on the cylinder $A \times \mathbb{R}$, with the properties listed below:

(i) $G$ is a continuous function defined on the set $\{(z, \zeta) \in(\bar{A} \times \mathbb{R}) \times(A \times \mathbb{R})$ : $z \neq \zeta\}$. Moreover, for every fixed $\zeta \in A \times \mathbb{R}, G(\cdot ; \zeta) \in \Gamma_{\text {loc }}^{2+\alpha}((A \times \mathbb{R}) \backslash\{\zeta\})$, and we have

$$
\mathcal{H}(G(\cdot ; \zeta))=0 \text { in }(A \times \mathbb{R}) \backslash\{\zeta\}, \quad G(\cdot ; \zeta)=0 \text { in } \partial A \times \mathbb{R} .
$$

(ii) We have $0 \leq G \leq \Gamma$. Moreover $G(x, t ; \xi, \tau)=0$ if $t \leq \tau$.

(iii) For every $\varphi \in C(\bar{A})$ such that $\varphi=0$ in $\partial A$ and for every fixed $\tau \in \mathbb{R}$, the function

$$
u(x, t)=\int_{A} G(x, t ; \xi, \tau) \varphi(\xi) \mathrm{d} \xi, \quad x \in \bar{A}, t>\tau,
$$

belongs to the class $\Gamma_{\mathrm{loc}}^{2+\alpha}(A \times(\tau, \infty)) \cap C(\bar{A} \times[\tau, \infty))$, and it solves

$$
\begin{cases}\mathcal{H} u=0 & \text { in } A \times(\tau, \infty), \\ u=0 & \text { in } \partial A \times[\tau, \infty), \\ u(\cdot, \tau)=\varphi & \text { in } \bar{A}\end{cases}
$$

Proof. For a fixed cylinder $D=A \times\left(T_{1}, T_{2}\right)$ we set

$$
h_{\zeta}^{D}(z)=\int \Gamma(\eta ; \zeta) \mathrm{d} \mu_{z}^{D}(\eta), \quad z, \zeta \in D,
$$

so that $G^{D}(z ; \zeta)=\Gamma(z ; \zeta)-h_{\zeta}^{D}(z)$ solves $\mathcal{H}\left(G^{D}(\cdot ; \zeta)\right)=0$ in $D \backslash\{\zeta\}, G^{D}(\cdot ; \zeta)=0$ in $\partial_{p} D$. Moreover, applying the weak maximum principle for $\mathcal{H}$ (see Theorem B) first in the set $A \times\left(T_{1}, \tau\right)$ and then in the set $A \times\left(\tau, T_{2}\right)$, we obtain

$$
G^{D}(x, t ; \xi, \tau)=0 \text { if } t \leq \tau, \quad G^{D}(x, t ; \xi, \tau) \geq 0 \text { if } t \geq \tau .
$$

We now consider the sequence $D_{n}=A \times(-n, n)$, and we observe that $G^{D_{n}}=G^{D_{n+1}}$ in $\left(D_{n} \cup \partial_{p} D_{n}\right) \times D_{n}$. This easily follows from (3.1) and from the weak maximum principle for $\mathcal{H}$ applied to the function $G^{D_{n}}(\cdot ; \zeta)-G^{D_{n+1}}(\cdot ; \zeta)=h_{\zeta}^{D_{n+1}}-h_{\zeta}^{D_{n}}$ in the set $A \times(\tau, n)$. As a consequence, the following definition of $G=G^{A}$ is well posed:

$$
G(z ; \zeta)=G^{D_{n}}(z ; \zeta), \quad \text { for } z \in D_{n} \cup \partial_{p} D_{n}, \quad \zeta \in D_{n} .
$$

Moreover, $G$ solves $\mathcal{H}(G(\cdot ; \zeta))=0$ in $(A \times \mathbb{R}) \backslash\{\zeta\}, G(\cdot, \zeta)=0$ in $\partial A \times \mathbb{R}$. We also define $h=h^{A}$ by

$$
h(z ; \zeta)=\Gamma(z ; \zeta)-G(z ; \zeta)
$$

(i.e., $h(z ; \zeta)=h_{\zeta}^{D}(z)$ for $\left.\zeta \in D=A \times\left(T_{1}, T_{2}\right), z \in D \cup \partial_{p} D\right)$. We claim that

$$
h \in C((\bar{A} \times \mathbb{R}) \times(A \times \mathbb{R})) .
$$

Since $h(\cdot ; \zeta)$ is continuous on $\bar{A} \times \mathbb{R}$, it is sufficient to prove that, for any $\zeta_{0} \in A \times \mathbb{R}$ and $T>0$,

$$
\sup _{z \in \bar{A} \times[-T, T]}\left|h(z ; \zeta)-h\left(z ; \zeta_{0}\right)\right| \longrightarrow 0, \quad \text { as } \zeta \rightarrow \zeta_{0} .
$$

Setting for brevity $D=A \times(-T, T)$ and $w_{\zeta}=h(\cdot ; \zeta)-h\left(\cdot ; \zeta_{0}\right), w_{\zeta}$ is a solution to $\mathcal{H} w_{\zeta}=0$ in $D, w_{\zeta}=\Gamma(\cdot ; \zeta)-\Gamma\left(\cdot ; \zeta_{0}\right)$ in $\partial A \times[-T, T], w_{\zeta}(\cdot,-T)=0$ in $\bar{A}$ (if $T$ is chosen large enough). Hence, by the weak maximum principle for $\mathcal{H}$,

$$
\sup _{z \in \bar{D}}\left|w_{\zeta}(z)\right| \leq \sup _{z \in \partial A \times[-T, T]}\left|\Gamma(z ; \zeta)-\Gamma\left(z ; \zeta_{0}\right)\right|
$$


which vanishes as $\zeta \rightarrow \zeta_{0}$, since $\Gamma$ is continuous away from the diagonal of $\mathbb{R}^{N+1} \times$ $\mathbb{R}^{N+1}$ (see Theorem $\mathrm{A}(\mathrm{i})$ ). Thus $(3.2)$ is proved, and the continuity of $G$ away from the diagonal of $(\bar{A} \times \mathbb{R}) \times(A \times \mathbb{R})$ immediately follows. In order to complete the proof of (i), it is only left to see that $G(\cdot ; \zeta) \in \Gamma_{\text {loc }}^{2+\alpha}((A \times \mathbb{R}) \backslash\{\zeta\})$. This will directly follow from Lemma 3.2 below.

Lemma 3.2. Let $\Omega$ be an open subset of $\mathbb{R}^{N+1}$. For every $u \in \Gamma^{2}(\Omega)$ such that $\mathcal{H} u=0$ in $\Omega$, we have $u \in \Gamma_{\text {loc }}^{2+\alpha}(\Omega)$ and

$$
\|u\|_{\Gamma^{2+\alpha}(\bar{O})} \leq \mathbf{c}(O, \Omega) \sup _{\Omega}|u|
$$

for every bounded domain $O \subset \bar{O} \subset \Omega$.

Proof. We refer to [12, 39]. See also [3, Proposition 4.6-Corollary 4.8].

Thus, the proof of (i) is completed. On the other hand, (ii) directly follows from (3.1) and by observing that $h \geq 0$ by the definition of $h_{\zeta}^{D}$. We now turn to the proof of (iii). We first prove the following lemma.

Lemma 3.3. Let $Y \in\left\{X_{i}, X_{i} X_{j}, \partial_{t} \mid i, j=1, \ldots, m\right\}$ and let $O, U$ be bounded domains of $\mathbb{R}^{N+1}$ such that $O \subset \bar{O} \subset U \subset \bar{U} \subset A \times \mathbb{R}$. We have

$$
\begin{gathered}
\|h(\cdot ; \zeta)\|_{\Gamma^{2+\alpha}(\bar{O})} \leq \mathbf{c}(O, U) \sup _{z \in U}|h(z ; \zeta)| \quad \forall \zeta \in A \times \mathbb{R}, \\
(z ; \zeta) \mapsto Y(h(\cdot ; \zeta))(z) \quad \text { is continuous in }(z ; \zeta) \in(A \times \mathbb{R}) \times(A \times \mathbb{R}), \\
\sup _{z \in \bar{O}, \zeta \in A \times[-T, T]}|Y(h(\cdot ; \zeta))(z)|<\infty .
\end{gathered}
$$

Proof. (3.3) straightforwardly follows from Lemma 3.2 recalling that $\mathcal{H}(h(\cdot ; \zeta))=0$ in $A \times \mathbb{R}$. Since $Y(h(\cdot ; \zeta))$ is continuous on $A \times \mathbb{R}$ for every fixed $\zeta \in A \times \mathbb{R}$, in order to prove (3.4) we only have to show that

$$
\sup _{z \in \bar{O}}\left|Y(h(\cdot ; \zeta))(z)-Y\left(h\left(\cdot ; \zeta_{0}\right)\right)(z)\right| \longrightarrow 0, \quad \text { as } \zeta \rightarrow \zeta_{0}
$$

for every fixed $\zeta_{0} \in A \times \mathbb{R}$ and every bounded domain $O \subset \bar{O} \subset A \times \mathbb{R}$. To this end, we apply Lemma 3.2 to the function $h(\cdot ; \zeta)-h\left(\cdot ; \zeta_{0}\right)$, and we obtain that the supremum in (3.6) is lower than

$$
\left\|h(\cdot ; \zeta)-h\left(\cdot ; \zeta_{0}\right)\right\|_{\Gamma^{2+\alpha}(\bar{O})} \leq \mathbf{c}(O, A) \sup _{z \in \bar{A} \times[-T(O), T(O)]}\left|h(z ; \zeta)-h\left(z ; \zeta_{0}\right)\right|,
$$

which vanishes as $\zeta \rightarrow \zeta_{0}$, by (3.2). This proves (3.4). Let us now prove (3.5). Let $O, U, V$ be bounded domains of $\mathbb{R}^{N+1}$ such that $O \subset \bar{O} \subset U \subset \bar{U} \subset V \subset \bar{V} \subset A \times \mathbb{R}$. Applying Lemma 3.2 to $h(\cdot ; \zeta)$ we get

$$
\begin{aligned}
& \sup _{z \in \bar{O}, \zeta \in(A \times[-T, T]) \backslash \bar{V}}|Y(h(\cdot ; \zeta))(z)| \leq \sup _{\zeta \in(A \times[-T, T]) \backslash \bar{V}}\|h(\cdot ; \zeta)\|_{\Gamma^{2+\alpha}(\bar{O})} \\
& \leq \mathbf{c}(O, U) \sup _{z \in U, \zeta \in(A \times[-T, T]) \backslash \bar{V}}|h(z ; \zeta)| \leq \mathbf{c}(O, U) \max _{z \in \bar{U}, \zeta \in(\bar{A} \times[-T, T]) \backslash V} \Gamma(z ; \zeta)<\infty,
\end{aligned}
$$

since $\Gamma$ is continuous away from the diagonal of $\mathbb{R}^{N+1} \times \mathbb{R}^{N+1}$. On the other hand, (3.4) directly yields $\sup _{z \in \bar{O}, \zeta \in \bar{V}}|Y(h(\cdot ; \zeta))(z)|<\infty$. Thus (3.5) is completely proved. 
We are now in position to prove (iii). Let $\varphi \in C(\bar{A}), \varphi=0$ in $\partial A$ and let $\tau \in \mathbb{R}$ be fixed. We agree to extend $\varphi$ to be zero outside $A$. Then, by means of Theorem $\mathrm{A}(\mathrm{iv})$, the function

$$
u_{1}(x, t)=\int_{\mathbb{R}^{N}} \Gamma(x, t ; \xi, \tau) \varphi(\xi) \mathrm{d} \xi, \quad x \in \mathbb{R}^{N}, t>\tau,
$$

belongs to the class $\Gamma_{\text {loc }}^{2+\alpha}\left(\mathbb{R}^{N} \times(\tau, \infty)\right) \cap C\left(\mathbb{R}^{N} \times[\tau, \infty)\right)$, and it is a solution to the Cauchy problem $\mathcal{H} u_{1}=0$ in $\mathbb{R}^{N} \times(\tau, \infty), u_{1}(\cdot, \tau)=\varphi$ in $\mathbb{R}^{N}$. On the other hand, recalling (3.2) and the fact that $|h| \leq \Gamma$, the function

$$
u_{2}(x, t)=\int_{A} h(x, t ; \xi, \tau) \varphi(\xi) \mathrm{d} \xi
$$

is well defined and continuous in $\bar{A} \times(\tau, \infty)$. Moreover, by means of Lemma 3.3, $u_{2}$ has continuous Lie derivatives up to second order along the vector fields $X_{1}, \ldots, X_{m}$, and continuous derivative along $\partial_{t}$, on $A \times(\tau, \infty)$, obtained by deriving under the integral sign. As a consequence, $\mathcal{H} u_{2}=0$ in $A \times(\tau, \infty)$. Furthermore, $u_{2} \in$ $\Gamma_{\text {loc }}^{2+\alpha}(A \times(\tau, \infty))$ by Lemma 3.2. Recalling that $G=\Gamma-h$ and that $u=u_{1}-u_{2}$, in order to complete the proof of Theorem 3.1 we are only left to show that

$$
u_{2}(x, t) \longrightarrow 0, \quad \text { as }(x, t) \rightarrow\left(x_{0}, \tau\right), \quad \forall x_{0} \in \bar{A} .
$$

If $x_{0} \in A$ we choose $\delta>0$ such that $B=\overline{B\left(x_{0}, \delta\right)} \subset A$ and we write

$$
\left|u_{2}(x, t)\right| \leq \int_{B} h(x, t ; \xi, \tau)|\varphi(\xi)| \mathrm{d} \xi+\int_{A \backslash B} h(x, t ; \xi, \tau)|\varphi(\xi)| \mathrm{d} \xi .
$$

Recalling (3.2) and the fact that $h(y, s ; \xi, \tau)=0$ for $s \leq \tau$, the first integral vanishes as $(x, t) \rightarrow\left(x_{0}, \tau\right)$; on the other hand, using the estimate $h \leq \Gamma$, the second integral goes to zero as well. Thus (3.7) holds for $x_{0} \in A$. If $x_{0} \in \partial A$, we use Theorem A(iv), and we obtain

$$
\left|u_{2}(x, t)\right| \leq \int_{\mathbb{R}^{N}} \Gamma(x, t ; \xi, \tau)|\varphi(\xi)| \mathrm{d} \xi \longrightarrow\left|\varphi\left(x_{0}\right)\right|=0, \quad \text { as }(x, t) \rightarrow\left(x_{0}, \tau\right) .
$$

This completes the proof.

Corollary 3.4. Let $A \subseteq \mathbb{R}^{N}$ be an $\mathcal{H}$-regular domain and let $G^{A}$ denote the related Green function as in Theorem 3.1. The following reproduction property of $G^{A}$ holds:

$$
G^{A}(x, t ; \xi, \tau)=\int_{A} G^{A}(x, t ; y, s) G^{A}(y, s ; \xi, \tau) \mathrm{d} y,
$$

for every $t>s>\tau$ and $x, \xi \in A$.

Proof. We fix $\xi, \tau, s$ as above, and we set $\varphi=G^{A}(\cdot, s ; \xi, \tau)$. Then $\varphi \in C(\bar{A}), \varphi=0$ in $\partial A$, by Theorem 3.1(i). Therefore we can apply Theorem 3.1(iii) and obtain that the function

$$
u(x, t)=\int_{A} G^{A}(x, t ; y, s) \varphi(y) \mathrm{d} y, \quad x \in \bar{A}, t>s,
$$

satisfies $u \in \Gamma_{\text {loc }}^{2+\alpha}(A \times(s, \infty)) \cap C(\bar{A} \times[s, \infty)), \mathcal{H} u=0$ in $A \times(s, \infty), u=0$ in $\partial A \times[s, \infty), u(\cdot, s)=\varphi$ in $\bar{A}$. It is now sufficient to observe that $G^{A}(\cdot ; \xi, \tau)$ has the same properties and to use the weak maximum principle for $\mathcal{H}$ (see Theorem B). 


\section{Estimates of the Green functions And Harnack inequality IN THE SMOOTH CASE}

Also in this section, we shall assume that the coefficients $a_{i, j}$ are smooth, as in Section 3. The following lemma (whose complete proof is given in 27]) states that it is always possible to make approximations by $\mathcal{H}$-regular domains of $\mathbb{R}^{N}$, both from the inside and from the outside. We recall that $d$ denotes the Carnot-Carathéodory control distance associated to $X_{1}, \ldots, X_{m}$.

Lemma 4.1. Let $B$ be a bounded open set of $\mathbb{R}^{N}$. Then for every $\delta>0$ there exist $\mathcal{H}$-regular domains $A^{\delta}, A_{\delta}$ such that $\{x \in B \mid d(x, \partial B)>\delta\} \subseteq A_{\delta} \subseteq B \subseteq A^{\delta} \subseteq\{x \in$ $\left.\mathbb{R}^{N} \mid d(x, \bar{B})<\delta\right\}$.

Sketch of the proof. The domain $A_{\delta}$ can be obtained as $B \backslash\left(\bigcup_{j=1}^{p} B_{d}\left(x_{j}, \delta\right)\right)$ where $x_{j} \in \partial B$ and $\left\{B_{d}\left(x_{j}, \delta\right)\right\}_{j}$ is a finite cover of $\partial B$. In this way, $A_{\delta}$ has the following property: there exists a positive constant $c$ such that $\left|B_{d}(x, r) \backslash A_{\delta}\right| \geq c\left|B_{d}(x, r)\right|$ for every $x \in \partial A_{\delta}$ and $r \in(0, \delta)$. In a similar way one can construct $A^{\delta}$ starting from the set $\left\{x \in \mathbb{R}^{N} \mid d(x, \bar{B})<\delta\right\}$, instead of $B$, and removing $d$-balls of radius $\delta$, centered at the boundary. The above construction of $A_{\delta}, A^{\delta}$ does not depend on $\mathcal{H}$ but only on the metric $d$. As a consequence such sets turn out to be $\mathcal{H}$-regular in a somewhat uniform way with respect to $\mathcal{H}$ (we shall use this fact in Section 5. see Lemma 5.1 below). The (non-trivial) details of this fact are given in the forthcoming paper [27, where we thoroughly investigate this topic.

We now fix $\delta_{0} \in(0,1)$. Then, by Lemma 4.1. for every $\xi_{0} \in \mathbb{R}^{N}$ and $R>0$, there exists a $\mathcal{H}$-regular domain $A\left(\xi_{0}, R\right)$ of $\mathbb{R}^{N}$ such that

$$
B_{d}\left(\xi_{0}, \delta_{0} R\right) \subseteq A\left(\xi_{0}, R\right) \subseteq B_{d}\left(\xi_{0}, R\right)
$$

Lemma 4.2. Let $R_{0}>0$ and $\delta \in\left(0, \delta_{0}\right)$. There exists a constant $\rho \in(0,1)$, only depending on $\delta, \delta_{0}, R_{0}$ and on the parameters $\Lambda, k, \alpha$ in (1.2) (and on the structure of $\mathbb{G})$, such that

$$
G^{A\left(\xi_{0}, R\right)}(x, t ; \xi, \tau) \geq \mathbf{c}\left(R_{0}\right)^{-1}(t-\tau)^{-Q / 2} \exp \left(-\mathbf{c} d^{2}(x, \xi) /(t-\tau)\right),
$$

for every $\xi_{0} \in \mathbb{R}^{N}, R \in\left(0, R_{0}\right], x \in A\left(\xi_{0}, R\right), \xi \in B_{d}\left(\xi_{0}, \delta R\right), t, \tau \in \mathbb{R}$, satisfying $d^{2}(x, \xi)<t-\tau<\rho R^{2}$.

Proof. Let $\xi_{0}, R, x, \xi, t, \tau$ be as above. We shall use the notation introduced in Section 3. Let us set $D=A\left(\xi_{0}, R\right) \times(\tau-1, t+1)$. Observing that

$$
\mu_{(x, t)}^{D}\left(\partial A\left(\xi_{0}, R\right) \times(t, t+1]\right)=0
$$

and using Theorem A(ii), we obtain

$$
\begin{aligned}
& h^{A\left(\xi_{0}, R\right)}(x, t ; \xi, \tau)=h_{(\xi, \tau)}^{D}(x, t)=\int_{\partial_{p} D} \Gamma(y, s ; \xi, \tau) \mathrm{d} \mu_{(x, t)}^{D}(y, s) \\
& \quad \leq \mathbf{c}\left(R_{0}\right) \int_{\partial A\left(\xi_{0}, R\right) \times[\tau, t]}(s-\tau)^{-Q / 2} \exp \left(-\frac{d^{2}(y, \xi)}{\mathbf{c}(s-\tau)}\right) \mathrm{d} \mu_{(x, t)}^{D}(y, s) \\
& \quad \leq \mathbf{c}\left(R_{0}\right) \sup _{0<r<t-\tau} r^{-Q / 2} \exp \left(-\frac{\left(\delta_{0}-\delta\right)^{2} R^{2}}{\mathbf{c} r}\right)
\end{aligned}
$$


(we have used the fact that $\xi \in B_{d}\left(\xi_{0}, \delta R\right)$ and that $\mu_{(x, t)}^{D}\left(\partial_{p} D\right)=1$ ). We now exploit Theorem 1.2, and we obtain

$$
\begin{aligned}
& G^{A\left(\xi_{0}, R\right)}(x, t ; \xi, \tau)=\Gamma(x, t ; \xi, \tau)-h^{A\left(\xi_{0}, R\right)}(x, t ; \xi, \tau) \\
& \geq \mathbf{c}_{1}\left(R_{0}\right)^{-1}(t-\tau)^{-Q / 2} \exp \left(-\mathbf{c} \frac{d^{2}(x, \xi)}{t-\tau}\right) \\
& \quad \times\left[1-\mathbf{c}_{2}\left(R_{0}\right) \sup _{0<r<t-\tau}\left(\frac{\rho R^{2}}{r}\right)^{Q / 2} \exp \left(-\frac{R^{2}}{\mathbf{c}\left(\delta, \delta_{0}\right) r}\right)\right] .
\end{aligned}
$$

It is now sufficient to observe that the expression between square brackets is greater than $1 / 2$ if $\rho=\rho\left(\delta, \delta_{0}, R_{0}\right)$ is small enough, as one can easily recognize by showing that the function $h \mapsto(\rho h)^{Q / 2} \exp \left(-h / \mathbf{c}\left(\delta, \delta_{0}\right)\right)$ is monotone decreasing on the interval $\left[\rho^{-1}, \infty\right)$.

Theorem 4.3. Let $R_{0}>0, T>1$ and $\gamma \in\left(0, \delta_{0}\right)$. We have

$$
G^{A\left(\xi_{0}, R\right)}(x, t ; \xi, \tau) \geq \mathbf{c}\left(T, \gamma, R_{0}, \delta_{0}\right)^{-1}(t-\tau)^{-Q / 2} \exp \left(-\mathbf{c}\left(\gamma, R_{0}, \delta_{0}\right) \frac{d^{2}(x, \xi)}{t-\tau}\right),
$$

for every $\xi_{0} \in \mathbb{R}^{N}, R \in\left(0, R_{0}\right], x, \xi \in B_{d}\left(\xi_{0}, \gamma R\right)$, and $0<t-\tau<T R^{2}$.

Proof. We set $\delta=\left(\gamma+\delta_{0}\right) / 2$, and we choose $\rho=\rho\left(\delta, \delta_{0}, R_{0}\right)$ as in Lemma 4.2. Let us also fix $\xi_{0}, R, x, \xi, t, \tau$ as above. Let $k$ be the smallest integer greater than $M\left(\gamma, \delta_{0}\right) \max \left\{T / \rho, d^{2}(x, \xi) /(t-\tau)\right\}$, where the constant $M\left(\gamma, \delta_{0}\right)>1$ will be chosen later, and let us set $\sigma=\frac{1}{4} \sqrt{(t-\tau) /(k+1)}$. We claim that there exists a chain of points of $\mathbb{R}^{N}, x=x_{0}, x_{1}, \ldots, x_{k+1}=\xi$, such that

$$
d\left(x_{j}, x_{j+1}\right) \leq \mathbf{c}\left(\gamma, \delta_{0}\right) \frac{d(x, \xi)}{k+1}, \quad d\left(x_{j}, \xi_{0}\right) \leq \frac{\gamma+\delta}{2} R .
$$

Indeed, if $d(x, \xi) \leq R\left(\delta_{0}-\gamma\right) / 4$, we can choose $x_{1}, \ldots, x_{k}$ laying on a suitable $X$-subunit path which gives a $d$-geodesic connecting $x$ and $\xi$, so that $d\left(x_{j}, x_{j+1}\right)=$ $d(x, \xi) /(k+1)$ and

$$
\begin{aligned}
d\left(x_{j}, \xi_{0}\right) & \leq d\left(x, x_{j}\right)+d\left(x, \xi_{0}\right) \leq d(x, \xi)+d\left(x, \xi_{0}\right) \\
& \leq R\left(\delta_{0}-\gamma\right) / 4+\gamma R=R(\delta+\gamma) / 2 .
\end{aligned}
$$

On the other hand, if $d(x, \xi)>R\left(\delta_{0}-\gamma\right) / 4$, then we can choose $x_{1}, \ldots, x_{k}$ laying on $d$-geodesics connecting $x$ with $\xi_{0}$ and $\xi_{0}$ with $\xi$, so that $d\left(x_{j}, x_{j+1}\right) \leq 2 \gamma R /(k+1)<$ $8 \gamma d(x, \xi)\left(\delta_{0}-\gamma\right)^{-1}(k+1)^{-1}$ and $d\left(x_{j}, \xi_{0}\right) \leq \gamma R$. Observing that, by the definition of $k$ and $\sigma$, we have $\sigma \leq M\left(\gamma, \delta_{0}\right)^{-1 / 2} R$, from (4.1) it follows that we can choose $M\left(\gamma, \delta_{0}\right)$ such that

$$
B_{d}\left(x_{j}, \sigma\right) \subseteq B_{d}\left(\xi_{0}, \delta R\right) .
$$

Moreover, up to a new choice of $M\left(\gamma, \delta_{0}\right)$, we also have

$$
d\left(y_{j}, y_{j+1}\right)<\sqrt{\frac{t-\tau}{k+1}} \quad \text { for every } y_{j} \in B_{d}\left(x_{j}, \sigma\right), y_{j+1} \in B_{d}\left(x_{j+1}, \sigma\right) .
$$

Indeed, from (4.1) and the definition of $k$ it follows that

$$
\begin{aligned}
d\left(y_{j}, y_{j+1}\right) & \leq 2 \sigma+d\left(x_{j}, x_{j+1}\right) \leq \frac{1}{2} \sqrt{\frac{t-\tau}{k+1}}+\mathbf{c}\left(\gamma, \delta_{0}\right) \frac{d(x, \xi)}{k+1} \\
& \leq \sqrt{\frac{t-\tau}{k+1}}\left(\frac{1}{2}+\mathbf{c}\left(\gamma, \delta_{0}\right) M\left(\gamma, \delta_{0}\right)^{-1 / 2}\right) .
\end{aligned}
$$


Now let $t=t_{0}, t_{1}, \ldots, t_{k+1}=\tau$ be such that $t_{j}-t_{j+1}=(t-\tau) /(k+1)$ for $j=0, \ldots, k$. Using Corollary 3.4 repeatedly, we obtain (we set $G=G^{A\left(\xi_{0}, R\right)}$, $\left.y_{0}=x, y_{k+1}=\xi\right)$

$$
\begin{aligned}
G(x, t ; \xi, \tau) & =\int_{\left(A\left(\xi_{0}, R\right)\right)^{k}} G\left(x, t ; y_{1}, t_{1}\right) G\left(y_{1}, t_{1} ; y_{2}, t_{2}\right) \cdots G\left(y_{k}, t_{k} ; \xi, \tau\right) \mathrm{d} y_{1} \cdots \mathrm{d} y_{k} \\
& \geq \int_{\prod_{j=1}^{k} B_{d}\left(x_{j}, \sigma\right)} \prod_{j=0}^{k} G\left(y_{j}, t_{j} ; y_{j+1}, t_{j+1}\right) \mathrm{d} y_{1} \cdots \mathrm{d} y_{k},
\end{aligned}
$$

by (4.2). Moreover, from (4.2), (4.3) and the definition of $k$, it follows that $y_{j+1} \in$ $B_{d}\left(\xi_{0}, \delta R\right), d^{2}\left(y_{j}, y_{j+1}\right)<(t-\tau) /(k+1)=t_{j}-t_{j+1}<T R^{2} /(k+1)<\rho R^{2}$. Therefore, we can apply Lemma 4.2 and obtain

$$
\begin{aligned}
& G(x, t ; \xi, \tau) \geq\left(\mathbf{c}\left(R_{0}\right)^{-1}\left(\frac{t-\tau}{k+1}\right)^{-\frac{Q}{2}}\right)^{k+1} \\
& \quad \times \int_{\prod_{j=1}^{k} B_{d}\left(x_{j}, \sigma\right)} \exp \left(-\mathbf{c} \sum_{j=0}^{k} \frac{d^{2}\left(y_{j}, y_{j+1}\right)}{t_{j}-t_{j+1}}\right) \mathrm{d} y_{1} \cdots \mathrm{d} y_{k} \\
& \quad \geq \mathbf{c}\left(R_{0}\right)^{-1}\left(\frac{t-\tau}{k+1}\right)^{-\frac{Q}{2}(k+1)}\left(\left|B_{d}(0,1)\right| \sigma^{Q}\right)^{k} \exp \left(-\mathbf{c}\left(R_{0}\right) k\right) \\
& \quad \geq \mathbf{c}\left(R_{0}\right)^{-1}(t-\tau)^{-\frac{Q}{2}} \exp \left(-\mathbf{c}\left(R_{0}\right) k\right),
\end{aligned}
$$

by the definition of $\sigma$. Now, if $d^{2}(x, \xi) \geq T(t-\tau) / \rho$, from the definition of $k$ it follows that $k<\mathbf{c}\left(\gamma, \delta_{0}\right) d^{2}(x, \xi) /(t-\tau)$ and then

$$
G(x, t ; \xi, \tau) \geq \mathbf{c}\left(R_{0}\right)^{-1}(t-\tau)^{-\frac{Q}{2}} \exp \left(-\mathbf{c}\left(\gamma, R_{0}, \delta_{0}\right) \frac{d^{2}(x, \xi)}{t-\tau}\right) .
$$

On the other hand, if $d^{2}(x, \xi)<T(t-\tau) / \rho$, the definition of $k$ gives $k<\mathbf{c}\left(\gamma, \delta_{0}\right) T / \rho$ and then

$$
G(x, t ; \xi, \tau) \geq \mathbf{c}\left(T, \gamma, R_{0}, \delta_{0}\right)^{-1}(t-\tau)^{-\frac{Q}{2}} .
$$

This completes the proof of Theorem 4.3 .

Lemma 4.4. Let $R_{0}>0$ and $\gamma \in\left(0, \delta_{0}\right)$. There exists a constant $\mu \in(0,1)$, $\mu=\mathbf{c}\left(\gamma, R_{0}, \delta_{0}\right)$, such that

$$
\frac{\operatorname{osc} u}{\overline{B_{d}\left(\xi_{0}, \gamma R\right)} \times\left[\tau_{0}-\gamma^{2} R^{2}, \tau_{0}\right]} \leq \mu \frac{\operatorname{osc} u}{B_{d}\left(\xi_{0}, R\right) \times\left[\tau_{0}-R^{2}, \tau_{0}\right]},
$$

for every $\left(\xi_{0}, \tau_{0}\right) \in \mathbb{R}^{N+1}, R \in\left(0, R_{0}\right]$ and every $u \in \Gamma^{2}\left(B_{d}\left(\xi_{0}, R\right) \times\left(\tau_{0}-R^{2}, \tau_{0}\right)\right) \cap$ $C\left(\overline{B_{d}\left(\xi_{0}, R\right)} \times\left[\tau_{0}-R^{2}, \tau_{0}\right]\right)$ satisfying $\mathcal{H} u=0$ in $B_{d}\left(\xi_{0}, R\right) \times\left(\tau_{0}-R^{2}, \tau_{0}\right)$.

Proof. We set $D=A\left(\xi_{0}, R\right) \times\left(\tau_{0}-R^{2}, \tau_{0}\right)$ and $S=\left\{x \in B_{d}\left(\xi_{0}, \gamma R\right) \mid u\left(x, \tau_{0}-R^{2}\right) \geq\right.$ $(M+m) / 2\}$, where $M=\max _{\bar{D}} u, m=\min _{\bar{D}} u$. We also define $w=u-m$ and (for $x \in \overline{A\left(\xi_{0}, R\right)}, t>\tau_{0}-R^{2}$ )

$$
v(x, t)=\int_{A\left(\xi_{0}, R\right)} G^{A\left(\xi_{0}, R\right)}\left(x, t ; y, \tau_{0}-R^{2}\right) w\left(y, \tau_{0}-R^{2}\right) \varphi(y) \mathrm{d} y,
$$

where $\varphi \in C_{0}\left(A\left(\xi_{0}, R\right)\right)$ is a cut-off function such that $0 \leq \varphi \leq 1$ and $\varphi \equiv 1$ in $B_{d}\left(\xi_{0}, \gamma R\right)$. By means of Theorem 3.1(iii), $v$ is a solution to $\mathcal{H} v=0$ in $D$, $v=0$ in $\partial A\left(\xi_{0}, R\right) \times\left[\tau_{0}-R^{2}, \tau_{0}\right], v\left(\cdot, \tau_{0}-R^{2}\right)=w\left(\cdot, \tau_{0}-R^{2}\right) \varphi$ in $\overline{A\left(\xi_{0}, R\right)}$. Therefore, by the weak maximum principle for $\mathcal{H}$ (see Theorem $\mathrm{B}$ ), $v \leq w$ in $D$. 
As a consequence, using the estimate in Theorem 4.3. for every $(x, t) \in D_{\gamma}=$ $B_{d}\left(\xi_{0}, \gamma R\right) \times\left(\tau_{0}-\gamma^{2} R^{2}, \tau_{0}\right)$ we get

$$
\begin{aligned}
w(x, t) & \geq v(x, t) \geq \int_{S} G^{A\left(\xi_{0}, R\right)}\left(x, t ; y, \tau_{0}-R^{2}\right)\left(\frac{M+m}{2}-m\right) \mathrm{d} y \\
& \geq \mathbf{c}\left(\gamma, R_{0}, \delta_{0}\right)^{-1} R^{-Q} \frac{M-m}{2}|S| .
\end{aligned}
$$

Now, if $|S| \geq \frac{1}{2}\left|B_{d}\left(\xi_{0}, \gamma R\right)\right|$, we infer that $\min _{\bar{D}_{\gamma}} u-m \geq \mathbf{c}\left(\gamma, R_{0}, \delta_{0}\right)^{-1}(M-m)$ and then

$$
\begin{aligned}
\operatorname{osc}_{\bar{D}_{\gamma}} u & \leq M-\frac{\min }{\bar{D}_{\gamma}} u \leq M-m-\mathbf{c}\left(\gamma, R_{0}, \delta_{0}\right)^{-1}(M-m) \\
& =\left(1-\mathbf{c}\left(\gamma, R_{0}, \delta_{0}\right)^{-1}\right) \operatorname{osc}_{\bar{D}} u .
\end{aligned}
$$

Recalling that $A\left(\xi_{0}, R\right) \subseteq B_{d}\left(\xi_{0}, R\right)$, we have proved (4.4) when $|S| \geq \frac{1}{2}\left|B_{d}\left(\xi_{0}, \gamma R\right)\right|$. On the other hand, if $|S|<\frac{1}{2}\left|B_{d}\left(\xi_{0}, \gamma R\right)\right|$, the argument above can be applied to $\widetilde{u}:=-u$, since (with the natural notation) $|\widetilde{S}|>\frac{1}{2}\left|B_{d}\left(\xi_{0}, \gamma R\right)\right|$. As a consequence, we get (4.4) for $\widetilde{u}$, and the proof is completed, since osc $\widetilde{u}=\operatorname{osc} u$.

Lemma 4.5. Let $R_{0}>0, h \in(0,1)$ and $\gamma \in\left(0, \delta_{0}\right)$. There exists a positive constant $\beta=\mathbf{c}\left(h, \gamma, R_{0}, \delta_{0}\right)$ such that

$$
\sup _{\lambda>0, s \in\left[\tau_{0}-R^{2}, \tau_{0}-h R^{2}\right]} \lambda \operatorname{meas}\left\{y \in B_{d}\left(\xi_{0}, \gamma R\right) \mid u(y, s) \geq \lambda\right\} \leq \beta R^{Q} u\left(\xi_{0}, \tau_{0}\right)
$$

for every $\left(\xi_{0}, \tau_{0}\right) \in \mathbb{R}^{N+1}, R \in\left(0, R_{0}\right]$ and every $u \in \Gamma^{2}\left(B_{d}\left(\xi_{0}, R\right) \times\left(\tau_{0}-R^{2}, \tau_{0}\right)\right) \cap$ $C\left(\overline{B_{d}\left(\xi_{0}, R\right)} \times\left[\tau_{0}-R^{2}, \tau_{0}\right]\right)$ satisfying $\mathcal{H} u=0, u \geq 0$ in $B_{d}\left(\xi_{0}, R\right) \times\left(\tau_{0}-R^{2}, \tau_{0}\right)$.

Proof. Let us fix $s \in\left[\tau_{0}-R^{2}, \tau_{0}-h R^{2}\right]$ and let us set $A=A\left(\xi_{0}, R\right)$ and

$$
w(x, t)=\int_{A} G^{A}(x, t ; y, s) u(y, s) \varphi(y) \mathrm{d} y, \quad x \in \bar{A}, t>s,
$$

where $\varphi \in C_{0}(A)$ is a cut-off function such that $0 \leq \varphi \leq 1$ and $\varphi \equiv 1$ in $B_{d}\left(\xi_{0}, \gamma R\right)$. By means of Theorem 3.1(iii), $w$ is a solution to $\mathcal{H} w=0$ in $A \times(s, \infty), w=0$ in $\partial A \times[s, \infty), w(\cdot, s)=u(\cdot, s) \varphi$ in $\bar{A}$. Therefore, by the weak maximum principle for $\mathcal{H}, w \leq u$ in $\bar{A} \times\left[s, \tau_{0}\right]$. As a consequence, using the estimate in Theorem 4.3 , we obtain

$$
u\left(\xi_{0}, \tau_{0}\right) \geq w\left(\xi_{0}, \tau_{0}\right) \geq \mathbf{c}\left(h, \gamma, R_{0}, \delta_{0}\right)^{-1} R^{-Q} \int_{B_{d}\left(\xi_{0}, \gamma R\right)} u(y, s) \mathrm{d} y .
$$

This ends the proof.

Theorem 4.6. Let $R_{0}>0,0<h_{1}<h_{2}<1$ and $\gamma \in\left(0, \delta_{0}\right)$. There exists a positive constant $M=\mathbf{c}\left(h_{1}, h_{2}, \gamma, R_{0}, \delta_{0}\right)$ such that

$$
\frac{\max _{\overline{B_{d}}\left(\xi_{0}, \gamma R\right)} \times\left[\tau_{0}-h_{2} R^{2}, \tau_{0}-h_{1} R^{2}\right]}{} u \leq M u\left(\xi_{0}, \tau_{0}\right)
$$

for every $\left(\xi_{0}, \tau_{0}\right) \in \mathbb{R}^{N+1}, R \in\left(0, R_{0}\right]$ and every $u \in \Gamma^{2}\left(B_{d}\left(\xi_{0}, R\right) \times\left(\tau_{0}-R^{2}, \tau_{0}\right)\right) \cap$ $C\left(\overline{B_{d}\left(\xi_{0}, R\right)} \times\left[\tau_{0}-R^{2}, \tau_{0}\right]\right)$ satisfying $\mathcal{H} u=0, u \geq 0$ in $B_{d}\left(\xi_{0}, R\right) \times\left(\tau_{0}-R^{2}, \tau_{0}\right)$.

Remark 4.7. Since $\delta_{0} \in(0,1)$ is arbitrary, the statement of Theorem 4.6 still holds true if $\delta_{0}$ is replaced by 1 . 
Proof of Theorem [4.6. Let $\mu=\mu\left(\frac{\delta_{0}}{2}, R_{0}, \delta_{0}\right) \in(0,1), \beta=\beta\left(h_{1}, \frac{\gamma+\delta_{0}}{2}, R_{0}, \delta_{0}\right)>0$ be as in Lemma 4.4 and Lemma 4.5 respectively. We define $r:(0, \infty) \rightarrow(0, \infty)$, $r(\lambda)=\left(\lambda(1-\mu)\left|B_{d}(0,1)\right| /(4 \beta)\right)^{-1 / Q}$, and we set $K=\left(1+\mu^{-1}\right) / 2$ and $M=$ $r^{-1}\left(\delta_{0}\left(1-h_{2}\right)\left(\delta_{0}-\gamma\right)\left(1-K^{-1 / Q}\right) / 4\right)$.

We now argue by contradiction and suppose that there exist $\xi_{0}, \tau_{0}, R$ and $u$ satisfying the hypotheses of the theorem, for which (4.5) is not true (with the above choice of $M)$. We first observe that $u\left(\xi_{0}, \tau_{0}\right) \neq 0$, since otherwise (4.5) would follow from Lemma 4.5. Now let $v=u / u\left(\xi_{0}, \tau_{0}\right)$. Since $v$ is bounded, in order to get a contradiction and thus prove the theorem, it is sufficient to show that there exists a sequence of points $\left\{\left(y_{j}, s_{j}\right)\right\}_{j \in \mathbb{N} \cup\{0\}}$ in $\overline{B_{d}\left(\xi_{0}, R\right)} \times\left[\tau_{0}-R^{2}, \tau_{0}\right]$ such that

$$
\begin{aligned}
& v\left(y_{j}, s_{j}\right) \geq K^{j} M, \quad\left(y_{j}, s_{j}\right) \text { belongs to the set } \\
& \begin{cases}\overline{B_{d}\left(\xi_{0}, \gamma R\right)} \times\left[\tau_{0}-h_{2} R^{2}, \tau_{0}-h_{1} R^{2}\right] & \text { if } j=0, \\
\overline{B_{d}}\left(y_{j-1}, 2 \delta_{0}^{-1} r\left(K^{j-1} M\right) R\right) \times\left[s_{j-1}-\left(2 \delta_{0}^{-1} r\left(K^{j-1} M\right) R\right)^{2}, s_{j-1}\right] & \text { if } j \geq 1 .\end{cases}
\end{aligned}
$$

Indeed, recalling that $K>1$, this would give $v\left(y_{j}, s_{j}\right) \rightarrow \infty$. Let us argue by induction. The existence of $\left(y_{0}, s_{0}\right)$ satisfying (4.6) for $j=0$ follows straightforwardly from the assumption that $u$ does not satisfy (4.5).

We now suppose that, for a fixed $q \in \mathbb{N} \cup\{0\},\left(y_{0}, s_{0}\right), \ldots,\left(y_{q}, s_{q}\right)$ have been defined and satisfy (4.6) for every $j \in\{0, \ldots, q\}$. We have to prove that we can find $\left(y_{q+1}, s_{q+1}\right)$ satisfying (4.6) for $j=q+1$. We claim that

$$
\overline{B_{d}}\left(y_{q}, 2 \delta_{0}^{-1} r\left(K^{q} M\right) R\right) \subseteq B_{d}\left(\xi_{0},\left(\gamma+\delta_{0}\right) R / 2\right) .
$$

Indeed, if $d\left(y, y_{q}\right) \leq 2 \delta_{0}^{-1} r\left(K^{q} M\right) R$, then recalling the definition of $M$ and using (4.6) for $j \in\{0, \ldots, q\}$, we obtain

$$
\begin{aligned}
d\left(y, \xi_{0}\right) & \leq d\left(\xi_{0}, y_{0}\right)+\sum_{j=1}^{q} d\left(y_{j-1}, y_{j}\right)+d\left(y_{q}, y\right) \\
& \leq \gamma R+2 \delta_{0}^{-1} R \sum_{i=0}^{q} r\left(K^{i} M\right)<\gamma R+2 \delta_{0}^{-1} r(M) R \sum_{i=0}^{\infty} K^{-i / Q} \\
& =\left(\gamma+\left(1-h_{2}\right)\left(\delta_{0}-\gamma\right) / 2\right) R<\left(\gamma+\delta_{0}\right) R / 2 .
\end{aligned}
$$

Moreover, with a similar computation we can prove that

$$
\left[s_{q}-\left(2 \delta_{0}^{-1} r\left(K^{q} M\right) R\right)^{2}, s_{q}\right] \subseteq\left(\tau_{0}-R^{2}, \tau_{0}-h_{1} R^{2}\right] .
$$

Indeed, $s_{q} \leq s_{q-1} \leq \cdots \leq s_{0} \leq \tau_{0}-h_{1} R^{2}$ and

$$
\begin{aligned}
& s_{q}-\left(2 \delta_{0}^{-1} r\left(K^{q} M\right) R\right)^{2}=s_{0}+\sum_{j=1}^{q}\left(s_{j}-s_{j-1}\right)-\left(2 \delta_{0}^{-1} r\left(K^{q} M\right) R\right)^{2} \\
& >\tau_{0}-h_{2} R^{2}-4 \delta_{0}^{-2}(r(M))^{2} R^{2} \sum_{i=0}^{\infty} K^{-2 i / Q} \\
& >\tau_{0}-\left(h_{2}+\left(1-h_{2}\right)\left(1-K^{-1 / Q}\right)\left(1+K^{-1 / Q}\right)^{-1}\right) R^{2}>\tau_{0}-R^{2} .
\end{aligned}
$$

We now apply Lemma 4.5 to $v$, and we obtain (recalling (4.8))

$$
\begin{gathered}
\operatorname{meas}\left\{y \in B_{d}\left(\xi_{0},\left(\gamma+\delta_{0}\right) R / 2\right) \mid v\left(y, s_{q}\right) \geq(1-\mu) K^{q} M / 2\right\} \\
\leq \frac{2 \beta R^{Q}}{(1-\mu) K^{q} M}<\operatorname{meas}\left\{B_{d}\left(y_{q}, r\left(K^{q} M\right) R\right)\right\} .
\end{gathered}
$$

As a consequence, since (4.7) also holds, there exists $\bar{y} \in B_{d}\left(y_{q}, r\left(K^{q} M\right) R\right)$ such that $v\left(\bar{y}, s_{q}\right)<(1-\mu) K^{q} M / 2$. Therefore, recalling that we are supposing that 
(4.6) holds for $j=q$, we have

$$
\begin{aligned}
& (1+\mu) K^{q} M / 2=K^{q} M-(1-\mu) K^{q} M / 2<v\left(y_{q}, s_{q}\right)-v\left(\bar{y}, s_{q}\right) \\
& \quad \leq \quad \operatorname{osc} v \quad \operatorname{osc} v \\
& \quad \underset{B_{d}\left(y_{q}, r\left(K^{q} M\right) R\right) \times\left\{s_{q}\right\}}{ } \leq \mu \frac{\overline{B_{d}}\left(y_{q}, 2 \delta_{0}^{-1} r\left(K^{q} M\right) R\right) \times\left[s_{q}-\left(2 \delta_{0}^{-1} r\left(K^{q} M\right) R\right)^{2}, s_{q}\right]}{}
\end{aligned}
$$

by means of Lemma 4.4. (4.7) and (4.8) (note that $2 \delta_{0}^{-1} r\left(K^{q} M\right) R \leq R_{0}$ by the definition of $M)$. Since $v \geq 0$, it follows that there exists $\left(y_{q+1}, s_{q+1}\right) \in$ $\overline{B_{d}}\left(y_{q}, 2 \delta_{0}^{-1} r\left(K^{q} M\right) R\right) \times\left[s_{q}-\left(2 \delta_{0}^{-1} r\left(K^{q} M\right) R\right)^{2}, s_{q}\right]$ such that

$$
v\left(y_{q+1}, s_{q+1}\right)>\mu^{-1}(1+\mu) K^{q} M / 2=K^{q+1} M .
$$

This completes the proof of Theorem 4.6

\section{HARNACK INEQUALITY IN THE NON-SMOOTH CASE}

In this section, we complete the proof of Theorem 1.1. Throughout the section the coefficients $a_{i, j}$ of $\mathcal{H}$ will not be supposed to be smooth as in Sections 34 above, but only Hölder continuous according to (1.2). We shall approximate $\mathcal{H}$ by suitable smooth coefficient operators $\mathcal{H}_{\varepsilon}$. Let $\Psi \in C_{0}^{\infty}\left(B_{d}(0,1) \times(-1,1)\right)$ be such that $0 \leq \Psi(x, t)=\Psi\left(x^{-1}, t\right), \int \Psi=1$ and set $\Psi^{\varepsilon}(x, t)=\varepsilon^{-Q-2} \Psi\left(\delta_{\varepsilon^{-1}} x, \varepsilon^{-2} t\right)$. We define

$$
a_{i, j}^{\varepsilon}(x, t)=\int_{\mathbb{R}^{N+1}} \Psi^{\varepsilon}\left(y \circ x^{-1}, s-t\right) a_{i, j}(y, s) \mathrm{d} y \mathrm{~d} s .
$$

Such a definition of regularization allows us to prove that the coefficients $a_{i, j}^{\varepsilon}$, besides being smooth, satisfy (1.2) (with the same $\Lambda, \alpha$ and $k$ ) and converge to $a_{i, j}$, uniformly on the compact subsets of $\mathbb{R}^{N+1}$, as $\varepsilon \rightarrow 0^{+}$.

Lemma 5.1. For every $\xi_{0} \in \mathbb{R}^{N}, R>0$ and $\delta \in(0,1)$, there exists a domain $A, \mathcal{H}_{\varepsilon}$-regular for any $\varepsilon>0$, such that $B_{d}\left(\xi_{0}, \delta R\right) \subseteq A \subseteq B_{d}\left(\xi_{0}, R\right)$ and with the following property: for any bounded cylinder $D=A \times\left(T_{1}, T_{2}\right)$ and for every $\varphi \in C\left(\partial_{p} D\right)$, letting $u_{\varepsilon}$ be the solution to $\mathcal{H}_{\varepsilon} u_{\varepsilon}=0$ in $D, u_{\varepsilon}=\varphi$ in $\partial_{p} D$, we have

$$
\left|u_{\varepsilon}(z)-\varphi\left(z_{0}\right)\right| \rightarrow 0, \quad \text { as } z \rightarrow z_{0} \text {, uniformly in } 0<\varepsilon<1 \text {, }
$$

for every $z_{0} \in \partial_{p} D$.

We refer to the proof of Lemma 4.1 for the construction of $A$. The (non-trivial) details of the proof of (5.2) are given in the forthcoming paper [27, along with a deeper potential theory study of the topic. Here, we only say that (5.2) is based on the construction of suitable uniform barrier functions.

Proof of Theorem 1.1. Chosen $\delta=\left(\max \left\{\gamma, h_{2}^{1 / 4}\right\}+1\right) / 2$, let $A$ be as in Lemma 5.1. We set $D=A \times\left(\tau_{0}-R^{2}, \tau_{0}\right), \varphi=\left.u\right|_{\partial_{p} D}$, and we denote by $u_{\varepsilon}$ the solution to $\mathcal{H}_{\varepsilon} u_{\varepsilon}=0$ in $D, u_{\varepsilon}=\varphi$ in $\partial_{p} D$. With this notation, (5.2) holds, thanks to Lemma 5.1. On the other hand, applying to $\mathcal{H}_{\varepsilon}$ the a priori estimates in Lemma 3.2 (we stress that any $a_{i, j}^{\varepsilon}$ satisfies (1.2) with the same $\Lambda, \alpha$ and $k$ ) and the maximum principle in Theorem B, we obtain

$$
\left\|u_{\varepsilon}\right\|_{\Gamma^{2+\alpha}(\bar{O})} \leq \mathbf{c}(O, D) \sup _{D}\left|u_{\varepsilon}\right| \leq \mathbf{c}(O, D) \max _{\partial_{p} D}|u|
$$

for every bounded domain $O \subset \bar{O} \subset D$. As a consequence, for some $\varepsilon_{k} \rightarrow 0, u_{\varepsilon_{k}}$ converges in $\Gamma_{\text {loc }}^{2+\alpha / 2}(D)$ to some function $v$ satisfying $\mathcal{H} v=0$ in $D$. From (5.2) and 
from the maximum principle for $\mathcal{H}$ it follows that it must be $v=u$. In particular $u_{\varepsilon_{k}}$ uniformly converges to $u$ on the compact subset of $D$. We now apply Theorem 4.6 (and Remark 4.7) to $u_{\varepsilon_{k}}$ (recall that $u_{\varepsilon} \geq 0$ since $\varphi \geq 0$ ) on the cylinder $B_{d}\left(\xi_{0}, R^{\prime}\right) \times\left(\tau_{\sigma}-R^{\prime 2}, \tau_{\sigma}\right)$, where $R^{\prime}=\delta R, \tau_{\sigma}=\tau_{0}-\sigma R^{\prime 2}$ and $\sigma>0$ is small. Setting $h_{2}^{\prime}=\sqrt{h_{2}}$ and $\gamma^{\prime}=\gamma / \delta$, we obtain

$$
\frac{\max _{\overline{B_{d}}\left(\xi_{0}, \gamma^{\prime} R^{\prime}\right)} \times\left[\tau_{\sigma}-h_{2}^{\prime} R^{\prime 2}, \tau_{\sigma}-h_{1} R^{\prime 2}\right]}{u_{\varepsilon_{k}}} \leq \mathbf{c}\left(h_{1}, h_{2}, \gamma, R_{0}\right) u_{\varepsilon_{k}}\left(\xi_{0}, \tau_{\sigma}\right) .
$$

First letting $k$ go to infinity and then $\sigma$ go to zero, from the above inequality we

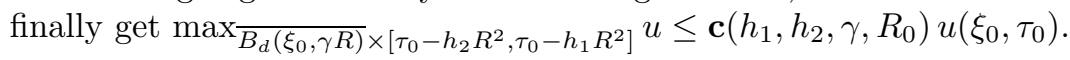

\section{ACKNOWLEDGEMENTS}

The authors would like to thank Professor E. Lanconelli for many useful conversations.

\section{REFERENCES}

1. G.K. Alexopoulos, Sub-Laplacians with drift on Lie groups of polynomial volume growth, Mem. Amer. Math. Soc. 155 (2002), no. 739. MR1878341(2003c:22015)

2. A. Bonfiglioli, E. Lanconelli, F. Uguzzoni, Uniform Gaussian estimates of the fundamental solutions for heat operators on Carnot groups, Adv. Differential Equations 7 (2002), 11531192. MR1919700 (2003f:35054)

3. A. Bonfiglioli, E. Lanconelli, F. Uguzzoni, Fundamental solutions for non-divergence form operators on stratified groups, Trans. Amer. Math. Soc. 356 (2004), 2709-2737. MR 2052194 (2005g:35037)

4. A. Bonfiglioli, F. Uguzzoni, Families of diffeomorphic sub-Laplacians and free Carnot groups, Forum Math. 16 (2004), 403-415. MR2050190 (2005f:22029)

5. A. Bonfiglioli, F. Uguzzoni, A note on lifting of Carnot groups, Rev. Mat. Iberoamericana 21 (2005), 1013-1035.

6. M. Bramanti, L. Brandolini, $L^{p}$ estimates for nonvariational hypoelliptic operators with VMO coefficients, Trans. Amer. Math. Soc. 352 (2000), 781-822. MR1608289 (2000c:35026)

7. M. Bramanti, L. Brandolini, $L^{p}$ estimates for uniformly hypoelliptic operators with discontinuous coefficients on homogeneous groups, Rend. Sem. Mat. Univ. Politec. Torino 58 (2000), 389-433. MR 1962808 (2004c:35058)

8. L. Capogna, Regularity of quasi-linear equations in the Heisenberg group, Comm. Pure Appl. Math. 50 (1997), no. 9, 867-889. MR1459590 (98k:22037)

9. L. Capogna, Regularity for quasilinear equations and 1-quasiconformal maps in Carnot groups, Math. Ann. 313 (1999), no. 2, 263-295. MR1679786 (2000a:35027)

10. L. Capogna, D. Danielli, N. Garofalo, An embedding theorem and the Harnack inequality for nonlinear subelliptic equations, Comm. Partial Differential Equations 18 (1993), no. 9-10, 1765-1794. MR1239930 (94j:35038)

11. L. Capogna, D. Danielli, N. Garofalo, Capacitary estimates and the local behavior of solutions of nonlinear subelliptic equations, Amer. J. Math. 118 (1996), no. 6, 1153-1196. MR.1420920 (97k:35033)

12. L. Capogna, Q. Han, Pointwise Schauder estimates for second order linear equations in Carnot groups, Proceedings for the AMS-SIAM Harmonic Analysis conference in Mt. Holyhoke, 2001.

13. G. Citti, N. Garofalo, E. Lanconelli, Harnack's inequality for sum of squares of vector fields plus a potential, Amer. J. Math. 115 (1993), 699-734. MR.1221840(94m:35069)

14. G. Citti, E. Lanconelli, A. Montanari, Smoothness of Lipschitz continuous graphs with nonvanishing Levi curvature, Acta Math. 188 (2002), 87-128. MR.1947459 (2003m:35035)

15. G. Citti, M. Manfredini, A. Sarti, A note on the Mumford-Shah functional in Heisenberg space, preprint. 
16. E. B. Fabes, D. W. Stroock, A new proof of Moser's parabolic Harnack inequality using the old ideas of Nash, Arch. Rational Mech. Anal. 96 (1986), 327-338. MR0855753 (88b:35037)

17. G. B. Folland, Subelliptic estimates and function spaces on nilpotent Lie groups, Ark. Mat. 13 (1975), 161-207. MR0494315(58:13215)

18. B. Franchi, G. Lu, R. L. Wheeden, Weighted Poincaré inequalities for Hörmander vector fields and local regularity for a class of degenerate elliptic equations, Potential Anal. 4 (1995), 361375. MR 1354890 (97e:35018)

19. P. Hajlasz, P. Koskela, Sobolev met Poincaré, Mem. Amer. Math. Soc. 145 (2000), no. 688. MR.1683160 (2000j:46063)

20. G. Huisken, W. Klingenberg, Flow of real hypersurfaces by the trace of the Levi form, Math. Res. Lett. 6 (1999), 645-661. MR1739222 (2001f:53141)

21. D. Jerison, J. M. Lee, The Yamabe problem on CR manifolds, J. Differential Geom. 25 (1987), 167-197. MR0880182 (88i:58162)

22. S. Kusuoka, D. Stroock, Applications of the Malliavin calculus III, J. Fac. Sci. Univ. Tokyo, Sect. IA, Math. 34 (1987), 391-442. MR0914028 (89c:60093)

23. S. Kusuoka, D. Stroock, Long time estimates for the heat kernel associated with a uniformly subelliptic symmetric second order operator, Ann. of Math. 127 (1988), 165-189. MR0924675 (89b:35022)

24. E. Lanconelli, Nonlinear equations on Carnot groups and curvature problems for CR manifolds, Renato Caccioppoli and modern analysis, Atti Accad. Naz. Lincei Cl. Sci. Fis. Mat. Natur. Rend. Lincei (9) Mat. Appl. 14 (2003), 227-238. MR2064269 (2005f:35048)

25. E. Lanconelli, A.E. Kogoj, $X$-elliptic operators and $X$-control distances, Contributions in honor of the memory of Ennio De Giorgi, Ricerche Mat. 49 (2000), suppl., 223-243. MR 1826225 (2002c:35121)

26. E. Lanconelli, A. Pascucci, S. Polidoro, Linear and nonlinear ultraparabolic equations of Kolmogorov type arising in diffusion theory and in finance, Nonlinear problems in mathematical physics and related topics, II, 243-265, Int. Math. Ser. (N.Y.), 2, Kluwer/Plenum, New York, 2002. MR 1972000 (2004c:35238)

27. E. Lanconelli, F. Uguzzoni, Cone criterion for non-divergence equations modeled on Hörmander vector fields, preprint.

28. G. Lu, Weighted Poincaré and Sobolev inequalities for vector fields satisfying Hörmander's condition and applications, Rev. Mat. Iberoamericana 8 (1992), 367-439. MR 1202416 (94c:35061)

29. G. Lu, Existence and size estimates for the Green's functions of differential operators constructed from degenerate vector fields, Comm. Partial Differential Equations 17 (1992), 12131251. MR 1179284 (93i:35030)

30. G. Lu, On Harnack's inequality for a class of strongly degenerate Schrödinger operators formed by vector fields, Differential Integral Equations 7 (1994), 73-100. MR1250940 (95f:35032)

31. A. Montanari, Real hypersurfaces evolving by Levi curvature: smooth regularity of solutions to the parabolic Levi equation, Comm. Partial Differential Equations 26 (2001), 1633-1664. MR.1865940 (2002i:35110)

32. R. Montgomery, A tour of subriemannian geometries, their geodesics and applications, Mathematical Surveys and Monographs 91, American Mathematical Society, Providence, RI, 2002. MR:1867362 (2002m:53045)

33. J. Petitot, Y. Tondut, Vers une neurogéométrie. Fibrations corticales, structures de contact et contours subjectifs modaux, Math. Inform. Sci. Humaines 145 (1999), 5-101. MR 1697185 (2000j:92007)

34. L. P. Rothschild, E. M. Stein, Hypoelliptic differential operators and nilpotent groups, Acta Math. 137 (1976), 247-320. MR0436223 (55:9171)

35. L. Saloff-Coste, D. W. Stroock, Opérateurs uniformément sous-elliptiques sur les groupes de Lie, J. Funct. Anal. 98 (1991), 97-121. MR1111195 (92k:58264)

36. Z. Slodkowski, G. Tomassini, Weak solutions for the Levi equation and envelope of holomorphy, J. Funct. Anal. 101 (1991), 392-407. MR.1136942 (93c:32018)

37. E. M. Stein, Harmonic analysis: real-variable methods, orthogonality, and oscillatory integrals, Princeton Mathematical Series 43, Princeton University Press, Princeton, NJ, MR.1232192(95c:42002) 
38. N. T. Varopoulos, L. Saloff-Coste, T. Coulhon, Analysis and geometry on groups, Cambridge Tracts in Mathematics 100, Cambridge University Press, Cambridge, 1992. MR 1218884 (95f:43008)

39. C. J. Xu, Regularity for quasilinear second-order subelliptic equations, Comm. Pure Appl. Math. 45 (1992), 77-96. MR1135924(93b:35042)

Dipartimento di Matematica, Università degli Studi di Bologna, Piazza di Porta S. Donato, 5 - 40126 Bologna, Italy

E-mail address: bonfigli@dm.unibo.it

Dipartimento di Matematica, Università degli Studi di Bologna, Piazza di Porta S. Donato, 5 - 40126 Bologna, Italy

E-mail address: uguzzoni@dm.unibo.it 